\title{
Comparison Analysis of CDMA Multiuser Detection using PSO and SMO
}

\author{
Amanpreet Kaur \\ Department of Yadavindra college of Engineering \\ Punjabi University \\ Patiala, India
}

\begin{abstract}
Using Spider monkey optimization (SMO) has become a common heuristic technique in many fields of engineering. In this paper, we apply SMO to solve near far effect problems in code division multiple access (CDMA) system, to reduce the computational complexity. The new approach is successfully tested in CDMA multiuser situation. The simulation results show that proposed algorithm is effective and outperforms as compare to methods like Particle Swarm Optimization (PSO) and Genetic Algorithm (GA).
\end{abstract}

\section{Keywords}

Spider Monkey Optimization; Particle Swarm Optimization; Genetic Algorithm; DS-CDMA multiuser detection.

\section{INTRODUCTION}

In recent years, the world has gone almost completely wireless. One type of wireless technology which has become very popular over the last few years is direct sequence code division multiple access (DS-CDMA). In this system, several users transmit information simultaneously over a common channel using pre-assigned signature codes called the spreading codes. But these codes are not correlate with each other. The performance of single user CDMA systems has restricted by multiple access interference (MAI), which if not controlled can severely degrades the quality of reception. In addition, the performance is limited by the presence of nearfar affect. The near-far problem is an important factor that affects the user capacity and performance of CDMA system [1].

To remove these problems, Multi-user detection (MUD) is considered as one of the key techniques for the 3rd Generation (3G) mobile communication system. This reduces the MAI and solves near-far problems and improves the capacity of system. The optimum detector is considered as best detector to reduce near far effect but its complexity increase exponentially by $2^{\mathrm{K}}$ where $\mathrm{K}$ is number of users.

Various optimization techniques are used to solve NP multiuser detection problems that reduce complexity of Optimum detector. One of the optimization algorithms is Spider monkey optimization algorithm is used as optimization tool in many fields of engineering. This algorithm is inspired by intelligent foraging behavior of fission- fusion social structure based animals [5].

This paper organized as follows: In Section II, we provide GA and PSO algorithm in detail. Then in Section III, social organization and behavior of spider monkey optimization, main steps of SMO Algorithm, Flow chart of SMO and its control parameters are illustrated. In Section IV, simulation results that compare the performance of different MUD algorithms are analyzed. Finally in Section V conclusion is presented.

\section{GA AND PSO ALGORITHM}

In this section, two evolutionary algorithms, namely Genetic Algorithm (GA), Particle Swarm Optimization Algorithm (PSO) will be discussed as the comparison group.

\section{A. Algorithm of $G A$}

$\mathrm{GA}$ is an optimization technique used to search a good answer for a problem. Chromosomes act as an individual in a system. This algorithm gives an idea about how these chromosomes communicate with a piece other and their atmosphere in a cooperative way to find a best solution for a problem that's why it is called optimization technique. It is categorized under an artificial intelligence because it is assumed that every individual pick and drop the data from one place to another in an artificial manner. First all the chromosomes are initialized with initial velocities and positions. After this the fitness value is calculated for each individual. The new fitness value of every individual is compared to previously known best fitness value. After this new position is calculated for each individual having fitness function better than earlier fitness value it is called personal best value. All the chromosomes know p-best value of their neighbors. The best p-best value is called g-best is calculated and this g-best value considered as best solution for whole population. This procedure continues till optimal value it calculated. This helps to find best way. The new population is generated by using cross over and mutation. This new population is considered as population for next iteration.

\section{B. Agorithm of PSO}

It is an optimization technique in which the particles act as individual in a system. This algorithm gives an idea about how these particles communicate with a piece other and their atmosphere in a cooperative way, to find a best solution to a problem that's why it is called optimization technique. It is categorized under artificial intelligence because It is assumed that every individual pick and drop the data from one place to another in an artificial manner. The main steps of algorithm are as shown below.

Step 1- It is assumed that number of particle is equal to number of bits.

Step 2- It is assumed that number of qualities in particle is equal to number of users.

Step 3- The food availability is equal to fitness value.

Step 4- The fitness function considered in one work is

$$
\mathrm{L}=-\left(\left(2 \times \mathrm{b}^{\prime} \times \mathrm{A} \times \mathrm{Y}\right)-\mathrm{b}^{\prime} \times \mathrm{A} \times \mathrm{R} \times \mathrm{b}\right)
$$

Step 5- Take maximum iteration $=100$

Step 6- Get the output from PSO stage and calculate BER between transmitted and received bits. 
Step 7- Vary value of SNR and repeat the steps to get BER for all the SNR values.

\section{SPIDER MONKEY OPTIMIZATION 3.1 Social organization and behavior}

SMO algorithm is based on the foraging behavior of spider monkeys. These monkeys fall in the category of fission fusion social structure based animals. They live in group of up to 50 individuals and break into small foraging groups that travel together and forage throughout the day. A female lead the group and responsible for searching the food source. In case she does not find the food then she divide the group into smaller groups that forage separately. The members of these subgroups communicate through barking and other physical activities within and outside the group depending on the availability of food.

C. Main Steps of Spider Monkey Algorithm (SMO)

1. Initialization of the population: Initially, SMO generates initial population of $\mathrm{N}$ spider monkeys where each monkey $\mathrm{SM}_{\mathrm{i}}$ is a D-dimensional vector. $\mathrm{D}$ is the no. of variables in the optimization problem and $\mathrm{SM}_{\mathrm{i}}$ represent the $i^{\text {th }}$ spider monkey in the population. Each $\mathrm{SM}_{\mathrm{i}}$ is initialized as follows:

$\mathrm{SM}_{\mathrm{i} j}=\mathrm{SM}_{\min \mathrm{j}}+\mathrm{U}(0,1) \times\left(\mathrm{SM}_{\max \mathrm{j}}-\mathrm{SM}_{\min \mathrm{j}}\right)$

2. Local Leader Phase (LLP): In this phase, each spider monkey SM modifies its current position based on the information of the local leader experience as well as local group member's experience. If the fitness value of the new position is higher than that of old position, then the SM updates his position. The position update equation for $\mathrm{i}^{\text {th }} \mathrm{SM}$ in this phase is:

$\mathrm{SMnew}_{\mathrm{ij}}=\mathrm{SM}_{\mathrm{ij}}+\mathrm{U}(0,1)\left(\mathrm{LL}_{\mathrm{kj}}-\mathrm{SM}_{\mathrm{ij}}\right)+\mathrm{U}(-1,1)\left(\mathrm{SM}_{\mathrm{r}}\right.$ j

$\left.\mathrm{SM}_{\mathrm{i}} \mathrm{j}\right)$

3. Global Leader Phase (GLP): After completion of the Local Leader Phase, the Global Leader Phase starts. In GLP phase, all the SM's modifies their position using experience of Global Leader and group member's experience. The position update equation is as follows :

$$
\begin{aligned}
& \mathrm{SMnew}_{\mathrm{ij}}=\mathrm{SM}_{\mathrm{ij}}+\mathrm{U}(0,1)\left(\mathrm{GL}_{\mathrm{j}}-\mathrm{SM}_{\mathrm{ij}}\right)+\mathrm{U}(-1,1)\left(\mathrm{SM}_{\mathrm{r} j}\right. \\
& \left.\mathrm{SM}_{\mathrm{i}} \quad \mathrm{j}\right)
\end{aligned}
$$$$
\text { (4) }
$$

In this phase, the positions of spider monkeys are updated based on probabilities prob $_{i}$ which are calculated using their fitness.

Prob $_{\mathrm{i}}=0.9$ fitness /max_fitness +0.1

4. Global Leader Learning (GLL) phase: In this phase, the position of the global leader is updated by applying the greedy selection in the population i.e., the position of the SM having best fitness in the population is selected as the updated position of the global leader. Further, it is checked that the position of global leader is updating or not and if not then the Global Limit Count is incremented by 1 .

5. Local Leader Learning (LLL) phase: In this phase, the position of the local leader is updated by applying the greedy selection in that group i.e., the position of the SM having best fitness in that group is selected as the updated position of the local leader. Next, the updated position of the local leader is compared with the old one and if the local leader is not updated then the Local Limit Count is incremented by 1 .

6. Local Leader Decision (LLD) phase: If any Local Leader position is not updated up to a predetermined threshold called Local Leader Limit, then all the members of that group update their positions either by random initialization or by using combined information from Global Leader and Local Leader, based on the pr.

$\underset{\left(\mathrm{SM}_{\mathrm{i}}\right.}{\mathrm{SMnew}_{\mathrm{ij}}}=\underset{\mathrm{j}}{\mathrm{SM}_{\mathrm{j}}}+\mathrm{U}(0,1) \times\left(\mathrm{GL}_{\mathrm{j}}-\mathrm{SM}_{\mathrm{ij}}\right)+\mathrm{U}(0,1) \times$ (5)

7. Global Leader Decision (GLD) phase: In this phase, the position of global leader is monitored and if it is not updated up to a predetermined number of iterations called Global Leader Limit, then the global leader divides the population into smaller groups. Firstly, the population is divided into two groups and then three groups and so on till the maximum number of groups (MG) are formed. Each time in GLD phase, LLL process is initiated to elect the local leader in the newly formed groups. The case in which maximum number of groups is formed and even then the position of global leader is not updated then the global leader combines all the groups to form a single group.

\section{CONTROL PARAMETERS IN SMO}

There are four control parameters in SMO algorithm

1. The value of Local Leader Limit should be $\mathrm{D} \times \mathrm{N}$

2. The value of Global Leader Limit should be $[N / 2$, $2 \times \mathrm{N}]$

3. $\mathrm{MG}=\mathrm{N} / 10$, i.e., it is chosen such that minimum number of SM's in a group should be 10

4. Perturbation rate should be $[0.1,0.9]$

Here $\mathrm{N}$ is the swarm size.

The flow chart of SMO algorithm is shown in figure 1. 


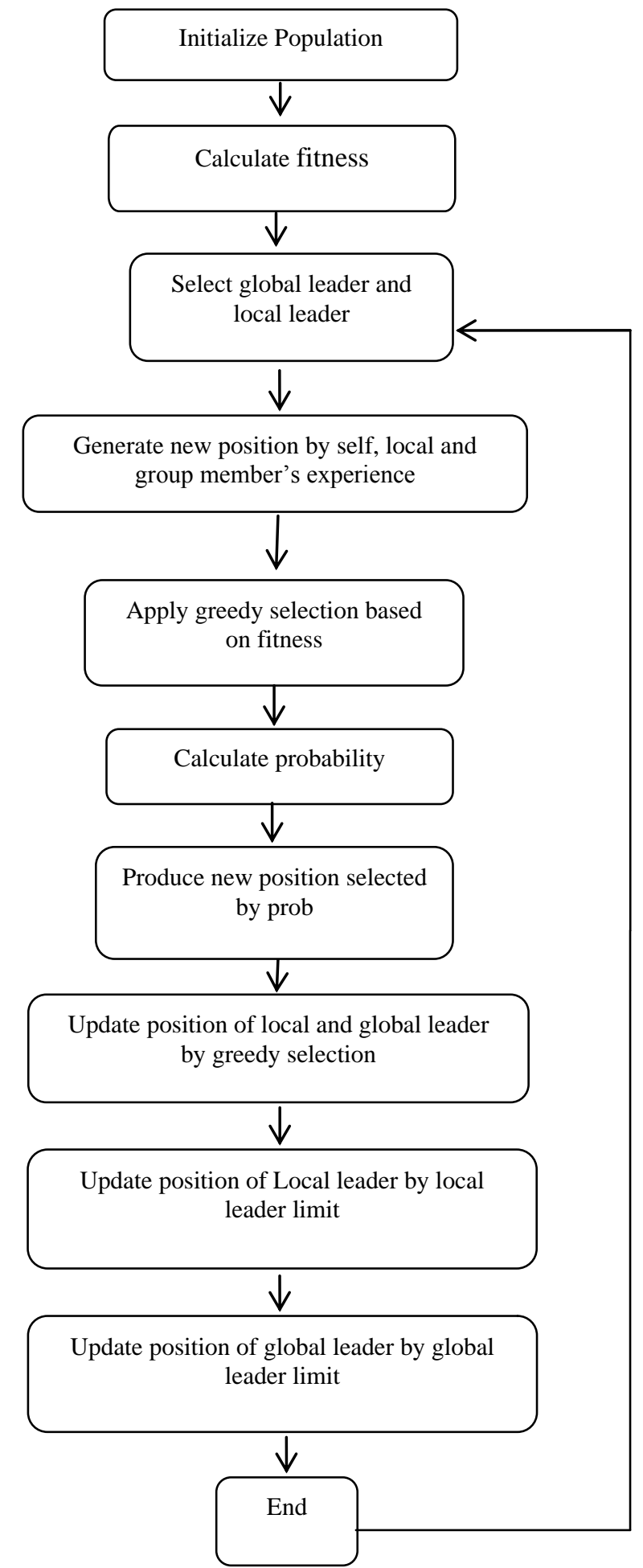

Figure 1. Flow chart of SMO

\section{RESULTS OF SIMULATION}

To test the performance of SMO it is compared with other two algorithms. The parameters used are bit error rate, signal to noise ratio, complexity and near far ratio. To simulate multiuser detection, 2-16 users CDMA system is considered. Gold codes have been used as spreading codes.

1. In the simulation system, $\mathrm{K}=16$, no. of bits $=10000$, modulation used is BPSK. An additive white Gaussian noise is used as a propagation channel and Rayleigh flat fading is also present. Under different SNR, the performance of GA, PSO and SMO has been shown figure 2 .

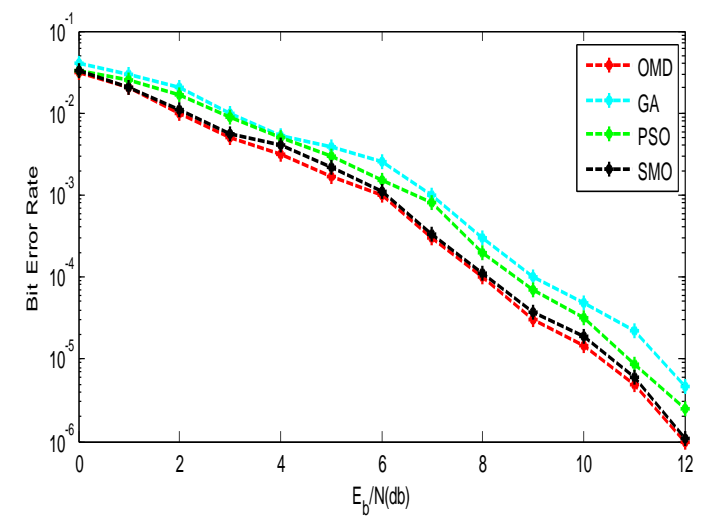

Figure 2. BER VS SNR

2. In figure 3 we compare BER performance against number of active users $\mathrm{K}$ at $\mathrm{SNR}=10 \mathrm{db}$ for CDMA system. It can be shown that SMO detector has a better BER performance than $\mathrm{PSO}$ at same $\mathrm{K}$.

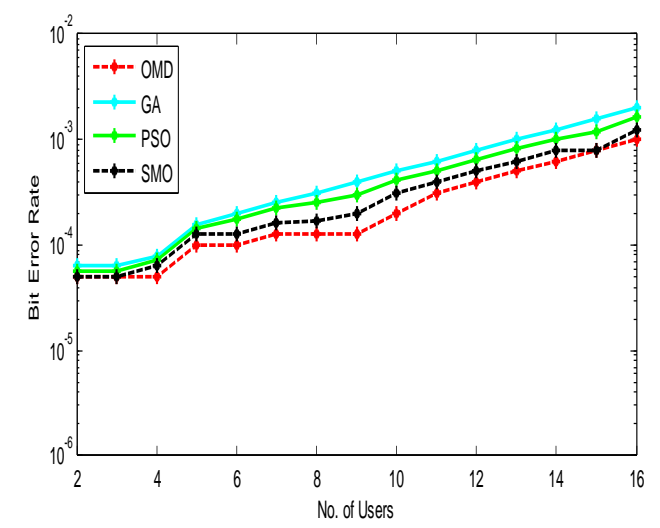

Figure 3. BER against the number of users

3. In figure 4, plots the BER performance of SMO and PSO detector against number of iterations. The number of iterations taken is 100 for simulation. The result shows that the complexity of SMO detector is lower than PSO.

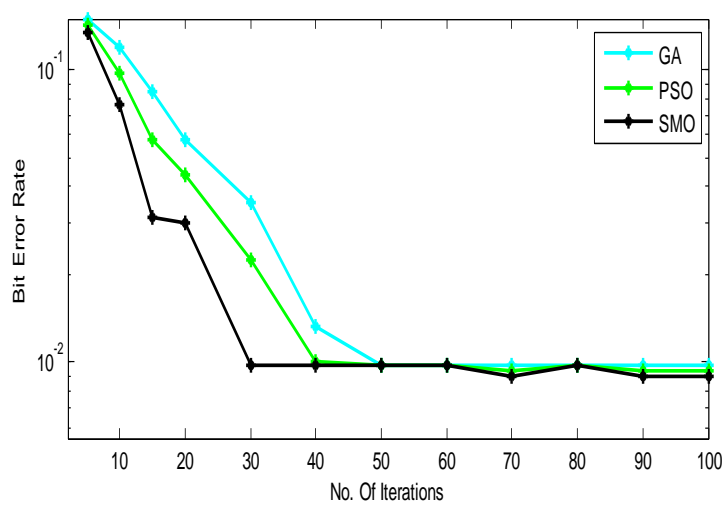

Figure 4. Complexity of SMO and PSO detector 
4. Figure 5 shows the variation of near far ratio with BER. It can be shown that BER performance of SMO is much better than PSO and GA.

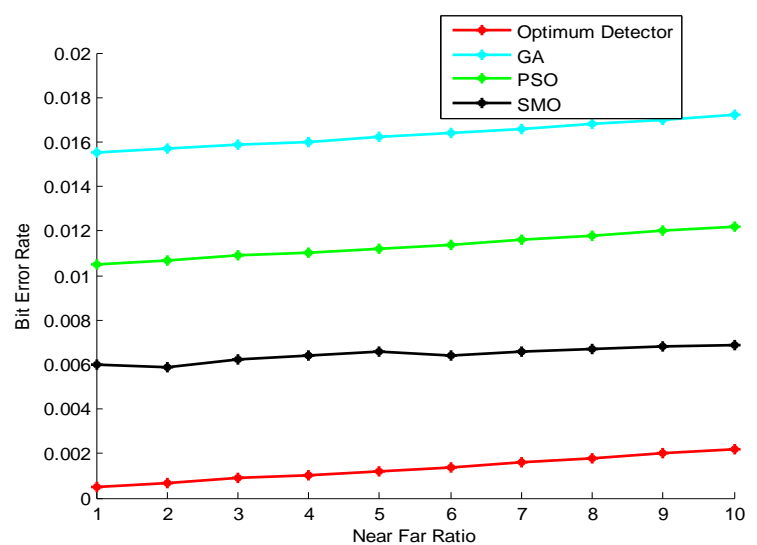

Figure 5. BER VS Near far ratio

\section{CONCLUSION}

In this paper, $\mathrm{SMO}$ is proposed as a strong candidate for near far problem. To verify its performance, it has been implemented in CDMA multiuser detection and compared with other evolutionary optimization methods. The results show the advantages of SMO in comparison to other methods as it can resist higher noise level, keep down BER and lower complexity. In this work SMO, PSO and GA algorithms have been used for the optimization and SMO performs much better than others. Furthermore other algorithms like Hybrid fire fly, Cuckoo search, Bat algorithm, Flower pollination have been proposed for future work. So there is a great scope of research in this area. Moreover, it is a valuable approach for real time multiuser detection in communication system.

\section{ACKNOWLEDGMENT}

This work has been done under the guidance of Mrs. Gurwinder Kaur, Assistant Professor, Section Electronics and Communication Engineering, Ycoe Department, Punjabi University Patiala that is gratefully acknowledged.

\section{REFERENCES}

[1] Atefeh Haji Jamali Arani and Paeiz Azmi, "Joint multiuser and inter symbol interference suppression in CDMA systems using particle swarm optimization algorithms," IEEE, pp. 978-983, 2013.

[2] Cong Wang, "A Complete Binary and HardwareEfficient PSO for CDMA Multiuser Detection," Sixth International Conference on Intelligent Human-Machine Systems and Cybernetics, pp. 3-6, 2014.

[3] J. G. Prokais, Digital Communications, New York: McGraw-Hill, 1989.

[4] J. Kennedy, and R. Eberhart, "Particle swarm optimization", in Proceedings of the IEEE International Conference on Neural Networks, vol. 4, pp. 1942-1948, December 1995.

[5] Jagdish Chand Bansal, Harish Sharma, Shimpi Singh Jadon and Maurice Clerc, "Spider Monkey Optimization algorithm for numerical optimization," Springer, Memetic Computing, pp. 31-47, 2014.

[6] Nacera Larbi, Fatima Debbat and A. Boudghene Stambouli, "A Review of Heuristic Near-Optimum MuD for MC-CDMA Systems," International Conference on Multimedia Computing and systems, pp. 1484-1489, 2014.

[7] Zhendong Yin, Xiaohui Liu, and Zhilu Wu, "A Multiuser Detector based on Artifical Bee Colony Algorithm for DS-UWB Systems," Hindawi Publishing Corporation, The Scientific World Journal, Article ID 547656, 8 pages, 2013 .

[8] Rapporteur, Theodore S. "Wireless Communications, Principles and Practice," Prentice-Hall, Inc. 2002.

[9] Siyere Sun and Weixiao Meng, "Uplink Pre equalization for CC-CDMA system under frequency selective fading," IEEE 2013 wireless communication symposium, pp. 5317-5321, 2013.

[10] Symington MMF, "Fission-fusion social organization inateles and pan. Int J Primatol vol. 11, no. 1, pp. 47-61, 1990 